\title{
Exercise by playing active video gaming in overweight and obese children
}

\author{
Han-Hung Huang ${ }^{1}$, Landon Cook ${ }^{1}$, Jessica Harrison ${ }^{1}$, Nicole O’Keefe $^{1}$, Kelsey Schultz ${ }^{1}$, Drew A \\ Curtis $^{2}$ \\ ${ }^{1}$ Department of Physical Therapy, Angelo State University, Texas Tech University System, 2601 W Ave N, San \\ Angelo, TX 76904, USA \\ ${ }^{2}$ Department of Psychology and Sociology, Angelo State University, Texas Tech University System, 2601 W Ave N, \\ San Angelo, TX 76904, USA
}

\begin{abstract}
Background: Active video gaming (AVG) has been suggested as a way of combating sedentary lifestyle in children. The objective was to investigate the effects of exercise by playing AVG on fitness and motivation for physical activity in children with overweight or obesity.

Methods: Fourteen children participated in an AVG program using Xbox Kinect and Nintendo Wii for one hour per session, two sessions per week for eight weeks. Three evaluations were performed at the beginning of the 1st, 9th and 16th sessions. The outcome measures include 1) physical fitness: vital signs, body mass index, body composition, muscular strength, muscular endurance, cardiovascular endurance and flexibility; 2) psychological assessments: Motives for Physical Activity MeasureRevised (MPAM-R) and Situational Motivational Scale (SIMS). In addition, real-time heart rates (HR) were monitored to assess exercise exertion throughout each session.

Results: No statistically significant changes were found in each component of physical and psychological assessments. MPAM-R was shown to have a good internal consistency reliability (Cronbach's alpha $>0.8$ ). Average and peak HR demonstrated the majority of subjects were able to achieve moderate or vigorous intensity of exercise during most AVG sessions.

Conclusions: Eight-week AVG program was not sufficient to show changes in fitness or motivation for physical activity in overweight and obese children. However, AVG might be an alternative and engaging way to reach moderate or vigorous intensity of exercise for this population. Future research may focus on a larger sample size and more frequent and longer training program to determine if benefits of AVG exist for overweight and obese children.
\end{abstract}

Keywords: Children, Overweight, Obese, Active video gaming, Fitness, Motivation.

Accepted on December 29, 2017

\section{Introduction}

The prevalence of overweight children and adolescents has more than tripled in the last three decades. In the United States, it is estimated that almost $16.2 \%$ of children and adolescents are overweight, with $17.2 \%$ being classified as obese [1]. Obesity in this population can lead to the development of a wide variety of physical and physiological problems such as orthopedic impairments, asthma, sleep apnea, hypertension and type 2 diabetes mellitus. High concentrations of liver enzymes associated with fatty liver, fatty hepatitis, fatty fibrosis or cirrhosis and increased formation of cholesterol that are commonly related to obesity in adults are now a frequent finding in obese children and adolescents [2,3]. Many of these associated co-morbidities that manifest in childhood can continue to plague the individual into adulthood and result in more serious complications including liver disease, atherosclerosis, metabolic syndrome, orthopedic complications, or even premature death. In addition, the impact of being overweight on the psychological mindset of a child is commonly understood. They are at a greater risk for developing depression, low self-esteem, poor body image, eating disorders, and behavior and learning problems [4-6]. They are likely to lead more sedentary lifestyles than their normal weight counterparts and less likely to participate due to feelings of insecurity and peer stigmatization [7].

It is crucial to balance physical activity and diet modification for overweight and obese children and adolescents. In simplest terms, more energy must be expended from physical activity than is taken in until a healthy weight is achieved. Eventually, a balance between the two must be maintained while young. Thus, the state of fitness can be extended into adulthood. Exercise is the one of the keys to affect the energy expenditure component of this equation [8]. Numerous studies have demonstrated the benefits of exercise in overweight and obese children and adolescents [9-11].

It has been estimated that children spend in an average of eight hours a week playing traditional sedentary video games $[12,13]$. Recently, active video games (AVG) have become a popular form of entertainment that requires limbs and body movement, coordination, as well as balance. Numerous systematic reviews and meta-analyses have suggested that AVG can be applied to combat a sedentary lifestyle in children [14-17]. One randomized trial found that incorporating AVG into pediatric weight management program has positive effects 
Citation: Huang HH, Cook L, Harrison J, et al.. Exercise by playing active video gaming in overweight and obese children. J Phys Ther Sports Med 2017;1(1):25-31.

on physical activity and body weight [18]. Another study applying AVG with exercise program in overweight and obese children identified improvements on body mass index (BMI) and weight circumference as well as self-esteem and psychological wellness state [19]. However, there is limited research looking into the changes of fitness levels and motivation for physical activity specifically in overweight and obese children after playing AVG. Therefore, the objective of the study was to investigate the effects of eight-week exercise programs by playing AVG on fitness levels including body composition, muscular strength, muscle endurance, cardiovascular endurance, and flexibility as well as psychological motivation for physical activity in children with overweight or obesity.

\section{Methods}

\section{Subjects}

A total of 14 subjects, between the ages of five and eighteen, were recruited via flyers, social media, and mass emails. The inclusion criteria were subjects who are able to participate without assistance, and are cognitively able to follow instructions for all activities. The exclusion criteria were subjects who had any orthopedic conditions, impaired mentation, or comorbidities that would contraindicate exercise training. A medical intake form was utilized to screen all participants for the presence of any exclusion criteria. Informed consent was obtained from both participants and their parent or legal guardian. This study was approved by the Angelo State University Institutional Review Board.

Each subject's BMI was calculated based on their height and weight which were recorded at the initial evaluation and classified based on their age and gender associated percentile rankings. In children and adolescents, percentiles are used as BMI is closely associated with developmental changes dependent upon gender and age [20]. Percentile rankings were calculated using the Centers for Disease Control BMI percentile calculator for child and teen [21]. The subjects were considered underweight if they were classified between $0-4 \%$, normal weight if they were classified between $5-84 \%$, and overweight if they were classified between $85-94 \%$, with an obese classification if greater than $95 \%$.

Ten subjects (eight males and two females) completed the study, with eight subjects' ethnicity reported as white and two as hispanic. Nine subjects BMI-for-age fell in the obese category, greater than the $95^{\text {th }}$ percentile, and one was overweight with BMI-for-age between the $85^{\text {th }}$ and $95^{\text {th }}$ percentile. Four subjects were unable to complete the final evaluation and were not included in the data analysis: one subject was unable to complete the study due to parental concern over the time the AVG program was taking out of his schoolwork, while another subject was unable to participate due to scheduling conflicts with baseball practice. The other two subjects dropped out before the final evaluation and did not provide a reason. The demographics for subjects who completed the study were summarized in Table 1.

Table 1. Demographics.

\begin{tabular}{llc}
\hline & Mean \pm SD & Range \\
\hline Age & $8.0 \pm 1.8$ & $5-10$ \\
\hline Height (cm) & $135.2 \pm 11.9$ & $120.0-158.7$ \\
\hline Body Fat (\%) & $36.2 \pm 5.4$ & $24.5-42.2$ \\
\hline Weight (Kg) & $45.8 \pm 11.0$ & $31.6-65.4$ \\
\hline BMI & $24.8 \pm 3.3$ & $20.0-30.6$
\end{tabular}

\section{Procedures}

The AVG program consisted of 15 gaming sessions among eight weeks with an average of two sessions per week. All sessions and evaluations were conducted by the research investigators. A total of three evaluations were performed over each eight-week period, with the first evaluation occurring at the first session, prior to gaming for that day. The midterm evaluation was performed at the ninth session, prior to gaming for that day and the final evaluation was performed during the 16 th session, with no gaming following. Each session consisted of a 5 minute warm-up, 50 minute gaming period, and a 5 minute cool down (see Appendix A for the order and duration of activities included in the warm-up and cool down). During the 50 minute gaming period, the subjects engaged in various AVG with one to two children per station (see Appendix B for the description of each AVG). The subjects were encouraged to engage in cooperative game play throughout all sessions. During each session, the subjects rotated gaming stations at least once. A total of two to three stations were available at each session. Various gaming consoles were utilized throughout the program consisting of the X-box 360 Kinect and Nintendo Wii.

\section{Assessments of motivation for physical activity}

Two subjective psychological outcome measures were completed upon arrival including the Motives for Physical Activity Revised (MPAM-R) and Situational Motivation Scale (SIMS) [22,23]. The subjects were instructed to answer honestly, and additional verbal instruction was offered by one of research investigators if the subjects' reading comprehension was limited due to age. The MPAM-R was applied to assess the subject's motivation for participating in 
physical activity. It consists of 30 questions assessing interest/ enjoyment, competence, appearance, fitness, and social reasons for the individual to participate in the study [22]. A 1-7 scale is used to answer each question with 1 being "Not at all true" and 7 being "Very true" (see Appendix C). The SIMS is a questionnaire consisting of sixteen total questions that assesses the individual's intrinsic and extrinsic motivation for exercise [23]. Similar to the MPAM-R, the SIMS also uses a 1-7 scale with 1 being "Not at all true" and 7 being "Very true" (see Appendix D).

\section{Assessments for fitness levels}

Following completion of the subjective psychological measures, the objective outcome measures for fitness levels were assessed. Objective outcomes measured at each evaluation in order of assessment included age, weight, height, heart rate, blood pressure, body composition, percent body fat, muscular strength, muscular endurance, cardiovascular endurance and flexibility. The outcomes were recorded to provide a baseline for each subject, to assess fitness involving different physical systems of the body, to see fitness improvements, and to ensure subjects met inclusion criteria.

The subject's age, weight, and height were all used to calculate the individual's BMI percentile. A single digital scale was used to assess each subject's weight. Height was assessed in standing with shoes off via a measuring tape mounted to the wall. Resting heart rate was assessed while seated using a pulse oximeter (Nonin Onyx 9590 Oximeter). An automatic blood pressure monitor (Carescape V100 Monitor, GE Healthcare) with an appropriate size cuff was used to assess resting blood pressure in sitting. Both heart rate and blood pressure were assessed while at rest to obtain a baseline for each subject's cardiovascular health.

The Omron handheld device (model HBF-306BL) was used to analyze each subject's body composition and percent body fat. Each subject's information was entered into the device including age, weight, height, and gender. This device had a minimal age cut-off at 10 years. For subjects below this age, 10 years was entered for their current age.

Muscular strength of the quadriceps and hamstrings was assessed via a handheld dynamometer (MicroFET 2). Examination of quadriceps strength was completed in a seated position in which the patient was cued to give maximal effort against the tester's resistance. Hamstring strength was assessed in a prone position and again the individual was cued for maximal effort. For both the quadriceps and hamstrings, an average of three max efforts was used as the reported score.

Muscular endurance was assessed by a one minute half sit-up test. During this test, the subject completed as many sit-ups as possible within a minute. Sit-ups were not counted if the subject did not reach the designated line while maintaining their hands on the ground.

Cardiovascular endurance was assessed via the twenty meter beep test. This test required the individual to run twenty meters at a set cadence until he or she was no longer able to keep up. The cadence gradually increased with each stage, and 7 stages were present in each level. Each participant was given one attempt to complete this test (see Appendix E for corresponding level of analysis).

Flexibility was the final measure assessed at each evaluation. A sit-and-reach box was used for all flexibility assessments. Testing technique required the subject to place their hands on top of one another and slowly lean forward as far as possible with shoes off. To prevent the subject's knees from bending, manual assistance by one of the research investigators was given at the knee. Three attempts were performed with the average recorded.

All measures described above were re-assessed at the midterm and final evaluation in the designated order.

\section{Exercise exertion monitoring during game play}

To monitor exercise exertion throughout the gaming session, heart rate was assessed and recorded, following warm-up and cool-down and at the completion of activities within each game via pulse oximeter placed on the participant's index finger. Timing between each assessment varied depending on the specific video game and activity within that game played.

The heart rate reserve (HRR) method was used to determine target exercise intensities, with the resting heart rate based on each subject's recorded resting heart rate from the initial evaluation for the first 8 sessions, and the midterm evaluation for the final 7 sessions (see Appendix F for HRR formula). Moderate intensity was determined based on $40-60 \%$ of the HRR as defined by the American College of Sports Medicine (ACSM), and vigorous intensity as greater than 60\% [24]. The average and peak heart rates of each participant were obtained from each session. They were used to determine the number of sessions in which the participants were able to maintain or achieve at least moderate intensity throughout each session.

\section{Statistical analysis}

Statistical analysis was completed using SPSS. A one-way repeated measures ANOVA was used to determine if there was any significant change in either the physical or psychological outcome measures. P-value of less than 0.05 was considered statistically significant. Reliability of the psychological scales, the MPAM-R and SIMS, was determined using Cronbach's alpha.

\section{Results}

\section{Physical fitness levels}

Over the eight-week AVG program, the improvements were noted on quadriceps muscle strength, muscle endurance tested by sit-ups and flexibility. However, the changes were not statistically significant. The body composition showed a slight improvement during the midterm of the program, but the effect did not maintain throughout the program. There was no 
Citation: Huang HH, Cook L, Harrison J, et al.. Exercise by playing active video gaming in overweight and obese children. J Phys Ther Sports Med 2017;1(1):25-31.

significant change on hamstrings muscles strength and cardiovascular endurance (Table 2).

Table 2. Physical fitness levels.

\begin{tabular}{|c|c|c|c|}
\hline & Initial & Midterm & Final \\
\hline Body Composition ( $\%$ of body fat) & $36.2 \pm 5.4$ & $35.4 \pm 5.0$ & $36.5 \pm 4.2$ \\
\hline Muscle Strength- Quadriceps (Ibs) & $37.4 \pm 8.4$ & $40.8 \pm 8.1$ & $40.8 \pm 7.3$ \\
\hline Muscle Strength- Hamstrings (Ibs) & $21.9 \pm 4.0$ & $20.6 \pm 3.5$ & $20.4 \pm 3.9$ \\
\hline Muscle Endurance (Number of sit-ups) & $25.0 \pm 11.7$ & $24.4 \pm 9.9$ & $25.7 \pm 9.4$ \\
\hline Cardiovascular Endurance (Stage of $20 \mathrm{~m}$ beep test) & $7.8 \pm 2.3$ & $7.4 \pm 1.8$ & $7.5 \pm 1.9$ \\
\hline Flexibility (cm) & $22.7 \pm 6.4$ & $23.6 \pm 5.8$ & $23.1 \pm 4.6$ \\
\hline
\end{tabular}

\section{Psychological motivation for physical activity}

On MPAM-R, the Cronbach's alpha coefficient ranged from 0.82 to 0.89 between three evaluations. While noting that a reliability coefficient of 0.80 or higher is considered "good" in most social science research [25], our data suggested that the responses from subjects have relatively high internal consistency in our study. However, there were no statistically significant changes on any category of assessment between the three evaluations (Table 3). On SIMS, the Cronbach's alpha coefficient was 0.61 and 0.69 at the initial and midterm evaluation respectively. At the final evaluation, the Cronbach's alpha was 0.50 indicating poor internal consistency. The results showed no significant changes on each category between the three evaluations (Table 4).

Table 3. Motives for Physical Activity Revised (MPAM-R).

\begin{tabular}{|c|c|c|c|}
\hline & Initial & Midterm & Final \\
\hline Interest and Enjoyment (Max=49) & $46.2 \pm 3.7$ & $44.5 \pm 5.8$ & $46 \pm 6.3$ \\
\hline Competence $(\operatorname{Max}=49)$ & $39.1 \pm 5.7$ & $40.5 \pm 5.0$ & $40.6 \pm 10.7$ \\
\hline Appearance $(\operatorname{Max}=42)$ & $28.0 \pm 7.1$ & $27.1 \pm 11.2$ & $27.5 \pm 9.4$ \\
\hline Fitness (Max=30) & $32.5 \pm 2.5$ & $31.7 \pm 4.0$ & $32.1 \pm 3.6$ \\
\hline Social $(\operatorname{Max}=30)$ & $25.4 \pm 6.5$ & $23.2 \pm 5.8$ & $24.5 \pm 7.5$ \\
\hline
\end{tabular}

Table 4. Situational motivation scale (SIMS).

\begin{tabular}{|c|c|c|c|}
\hline & Initial & Midterm & Final \\
\hline Intrinsic Motivation $(\max =28)$ & $27.0 \pm 1.2$ & $26.1 \pm 3.0$ & $25.8 \pm 3.0$ \\
\hline Identified Regulation $(\max =28$ ) & $24.2 \pm 4.1$ & $23.5 \pm 4.5$ & $23.8 \pm 3.1$ \\
\hline External Regulation $(\min =0$ ) & $9.5 \pm 4.3$ & $10.9 \pm 7.8$ & $9.8 \pm 7.1$ \\
\hline Amotivation $(\min =0)$ & $8.8 \pm 5.5$ & $7.2 \pm 3.3$ & $8.1 \pm 3.9$ \\
\hline
\end{tabular}

\section{Discussion}

The objective of this study was to assess the effects of eightweek AVG program on fitness parameters and motivational factors for physical activity in overweight and obese children. Previous studies demonstrated that youth AVG players can obtain some light- to moderate- intensity physical activity $[14,25,26]$. One recent study suggested that healthy teens and children were able to reach moderate to vigorous intensity by playing AVG with X-box 360 Kinect [27]. In our study, overweight and obese children were playing X-box 360 Kinect and/or Nintendo Wii during 1 hour session for 8 weeks. Our results of exercise exertion when playing AVG were in agreement with existing literature. Based on each subject's average heart rates during each session, four subjects were able to achieve moderate intensity physical activity on the majority of sessions during the study. Three subjects were able to achieve moderate levels of intensity occasionally but stayed at the light intensity level for most of the sessions. The remaining three subjects averaged only light intensity physical activity during all 15 sessions. At certain time points, almost every subject ( 9 out of 10) was able to reach moderate or even vigorous intensity physical activity levels based on their peak heart rates during each session. 
Previous research investigated the effects of AVG on weight loss or BMI in overweight and obese children population $[18,19]$. In this study, we focused on fitness measures including body composition, muscular strength, muscle endurance, cardiovascular endurance and flexibility because of their clinical significance regarding overall health in children and adolescents [28,29]. Our results showed that there was no statistically significant change in the fitness levels after 8 week AVG program. Insufficient session frequency and program duration may explain the lack of significant changes. For healthy children, at least 60 minutes of intermittent moderate to vigorous-intensity exercise daily is recommended by World Health Organization and US Department of Health and Human Services [30,31]. ACSM recommended that children and adolescents need to participate in physical activity for 60 minutes per day with a minimum of three days per week at vigorous intensity for health [24]. However, the consensus on the ideal frequency and duration to perform AVG as a mean of physical activity to reach desired health benefits has not been established. Therefore, we suggest future study with a more frequent (at least 3 days per week) and longer training program (more than 2 months) is required to corroborate the effects of AVG on physical fitness for overweight and obese children.

The MPAM-R was selected to assess subject's motivation for participation in physical activity, playing AVG in this study. Our data showed good reliability with a high internal consistency, even though the changes were not significant. Current literature supports the use of MPAM-R within a variety of adult populations as a tool to assess motivation and projected exercise adherence [32-35]. The psychometric properties in adult was originally reported by Ryan and colleagues in 1997 [22]. Recently, one study reported the reliability of MPAM-R was $0.77-0.79$ in fifth (age 10.5 \pm 0.5 ) and sixth (age $11.5 \pm 0.5$ ) grades boys and girls [36]. To our best knowledge, we are the first group reporting the reliability in overweight and obese children population between age 5 and 10 (age $8.0 \pm 1.8$ ). The SIMS, similar to the MPAM-R, was selected to assess each subject's intrinsic and extrinsic motivation. The SIMS has been applied in several studies with various age populations from youth students (with age of 12 and above) to adult [37-42]. The reliability and validity of SIMS has been reported in physical education context [43]. However, the results in our study did not produce scores with acceptable reliability and significant changes. We suggested the average age of our subjects and the different setting compared to previous studies might play a role here. In addition, while we did not find statistically significant differences in motivational measures, it is important to note that scores on motivation were high prior to beginning the activity. This ceiling effect might be attributed to excitement of the subjects with an early peak in motivation. Because of the ceiling effect, there was little to no room for a significant positive change.

A number of limitations were recognized in this study. First, the sample size was small and four of 14 subjects did not participate in the final evaluation. Second, we did not control subjects' diet or restrict to other physical activities during the eight-week study. In addition, we noticed that some subjects' degree of effort fluctuated during AVG and it depended on who the subject was paired with during play. We also recognized that some subjects lost interest in engaging in the assessment activities due to the frequency of the evaluations in this short period of study.

In conclusion, exercise by playing AVG twice per week for eight weeks did not significantly change fitness or motivation for physical activity in overweight and obese children. MPAM$\mathrm{R}$ showed a good reliability which was reported for the first time in this specific young population. Future study with a larger sample size, more frequent and longer training program is required to determine the benefits of $A V G$ for this population.

\section{Clinical significance}

AVG might be an alternative and engaging way to reach moderate or vigorous intensity of exercise for overweight and obese children. It can be tailored based on the individual's interests and preferred styles of exercise through the numerous gaming options. In addition, AVG has the potential to be utilized at the health clinic, weight loss program, school and home environment to benefit this population.

\section{Disclosure Statement}

No competing financial interests exist.

\section{References}

1. Fryar CD, Carroll MD, Ogden CL. Prevalence of Overweight and Obesity Among Children and Adolescents Aged 2-19 Years: United States, 1963-1965 Through 2013-2014. National Center for Health Statistics: Division of Health and Nutrition Examination Surveys. 2016.

2. Dietz WH. Health consequences of obesity in youth: childhood predictors of adult disease. Pediatr. 1998;101(S2):518-25.

3. Reilly JJ, Methven E, McDowell ZC, et al. Health consequences of obesity. Arch Dis Child. 2003;88(9): 748-52.

4. Krebs NF, Jacobson MS. Prevention of pediatric overweight and obesity. Pediatr. 2003;112(2):424-30.

5. Pagoto SL, Curtin C, Lemon SC, et al. Association between adult attention deficit/hyperactivity disorder and obesity in the US population. Obesity (Silver Spring). 2009;17(3): 539-44.

6. Pagoto SL, Schneider KL, Bodenlos JS, et al. Association of post-traumatic stress disorder and obesity in a nationally representative sample. Obesity (Silver Spring). 2012;20(1): 200-5.

7. Stankov I, Olds T, Cargo M. Overweight and obese adolescents: what turns them off physical activity? Int J Behav Nutr Phys Act. 2012;9:53.

8. Spear BA, Barlow SE, Ervin C, et al. Recommendations for treatment of child and adolescent overweight and obesity. Pediatr. 2007;120(S4):S254-88. 
Citation: Huang HH, Cook L, Harrison J, et al.. Exercise by playing active video gaming in overweight and obese children. J Phys Ther Sports Med 2017;1(1):25-31.

9. Wang $\mathrm{Y}$, Wu Y, Wilson RF, et al. Childhood Obesity Prevention Programs: Comparative Effectiveness Review and Meta-Analysis. Rockville MD, USA. 2013.

10. Ho M, Garnett SP, Baur LA, et al. Impact of dietary and exercise interventions on weight change and metabolic outcomes in obese children and adolescents: a systematic review and meta-analysis of randomized trials. JAMA Pediatr. 2013;167(8):759-68.

11. Vale S, Trost S, Ruiz JJ, et al. Physical activity guidelines and preschooler's obesity status. Int J Obes (2005). 2013;37(10):1352-5.

12. Generation M2: Media in the Lives of 8- to 18-Year-Olds. Henry J. Kaiser Family Foundation, California, US. 2010.

13. Christakis DA, Ebel BE, Rivara FP, et al. Television, video, and computer game usage in children under 11 years of age. J Pediatr. 2004;145(5):652-6.

14. LeBlanc AG, Chaput JP, McFarlane A, et al. Active video games and health indicators in children and youth: a systematic review. PloS One. 2013;8(6):e65351.

15. Peng W, Crouse JC, Lin JH. Using active video games for physical activity promotion: a systematic review of the current state of research. Health education \& behavior: The official publication of the Society for Public Health Education. 2013;40(2):171-192.

16. Gao Z, Chen S, Pasco D, et al. A meta-analysis of active video games on health outcomes among children and adolescents. Obes Rev. 2015;16(9):783-94.

17. Lamboglia CM, da Silva VT, de Vasconcelos Filho JE, et al. Exergaming as a strategic tool in the fight against childhood obesity: a systematic review. J Obes. 2013;2013:438364.

18. Trost SG, Sundal D, Foster GD, et al. Effects of a pediatric weight management program with and without active video games a randomized trial. JAMA Pediatr. 2014;168(5): 407-13.

19. Duman F, Kokacya MH, Dogru E, et al. The Role of Active Video-Accompanied Exercises in Improvement of the Obese State in Children: A Prospective Study from Turkey. J Clin Res Pediatr Endocrinol. 2016;8(3):334-40.

20. Centers for Disease Control and Prevention. About Child \& Teen BMI. 2015.

21. Centers for Disease Control and Prevention. BMI Percentile Calculator for Child and Teen English Version.

22. Ryan RM, Frederick CM, Lepes D, et al. Intrinsic motivation and exercise adherence. Int J Sport Psychol. 1997;28(4):335-54.

23. Guay F, Vallerand RJ, Blanchard C. On the Assessment of Situational Intrinsic and Extrinsic Motivation: The Situational Motivation Scale (SIMS). Motiv Emot. 2000;24(3):175-213.

24. Pescatello LS. American College of Sports M. ACSM's guidelines for exercise testing and prescription. 9th ed. 2014. J Can Chiropr Assoc. 2014;58(3):328.

25. Cronbach LJ. Coefficient alpha and the internal structure of tests. Psychometrika. 1951;16(3):297-334.
26. O'Donovan C, Roche EF, Hussey J. The energy cost of playing active video games in children with obesity and children of a healthy weight. Pediatr Obes. 2014;9(4): 310-7.

27. Clevenger KA, Howe CA. Energy Cost and Enjoyment of Active Videogames in Children and Teens: Xbox 360 Kinect. Games Health J. 2015;4(4):318-24.

28. Ortega FB, Ruiz JR, Castillo MJ, et al. Physical fitness in childhood and adolescence: a powerful marker of health. Int J Obes (2005). 2008;32(1):1-11.

29. Janssen I, Leblanc AG. Systematic review of the health benefits of physical activity and fitness in school-aged children and youth. Int J Behav Nutr Phys Act. 2010;7:40.

30. World Health Organization. Global Recommendations on Physical Activity for Health (5-17 years old). World Health Organization. 2011.

31. Buchner DM, Bishop J, Brown DR, et al. Physical Activity Guidelines for Americans. In: US Department of Health and Human Services (ed). 2008.

32. Kane I, Lee H, Sereika S, et al. Feasibility of pedometers for adults with schizophrenia: pilot study. J Psychiatr Mental Health Nurs. 2012;19(1):8-14.

33. Sibley BA, Hancock L, Bergman SM. University students exercise behavioral regulation, motives, and physical fitness. Percept Mot Skills. 2013;116(1):322-39.

34. Albuquerque MR, Lopes MC, de Paula JJ, et al. CrossCultural Adaptation and Validation of the MPAM-R to Brazilian Portuguese and Proposal of a New Method to Calculate Factor Scores. Front Psychol. 2017;8:261.

35. Withall J, Jago R, Fox KR. Why some do but most don't? Barriers and enablers to engaging low-income groups in physical activity programmes: a mixed methods study. BMC Public Health. 2011;11:507.

36. Dishman RK, Saunders RP, McIver KL, et al. Construct validity of selected measures of physical activity beliefs and motives in fifth and sixth grade boys and girls. J Pediatr Psychol. 2013;38(5):563-76.

37. Gao Z, Hannon JC, Newton M, et al. Effects of curricular activity on students' situational motivation and physical activity levels. Res Q Exerc Sport. 2011;82(3):536-44.

38. Jaakkola T, Liukkonen J, Laakso T, et al. The relationship between situational and contextual self-determined motivation and physical activity intensity as measured by heart rates during ninth grade students' physical education classes. Eu Phys Educ Rev. 2008;14(1):13-31.

39. Lonsdale C, Sabiston CM, Raedeke TD, et al. Selfdetermined motivation and students' physical activity during structured physical education lessons and free choice periods. Prev Med. 2009;48(1):69-73.

40. Nicaise V, Kahan D. Psychological changes among Muslim students participating in a faith-based school physical activity program. Res Q Exerc Sport. 2013;84(4):522-9.

41. Escher C, Creutzfeldt J, Meurling L, et al. Medical students' situational motivation to participate in simulation based team training is predicted by attitudes to patient safety. BMC Med Educ. 2017;17(1):37. 
42. Naisseh M, Martinent G, Ferrand C, et al. Relationship Between Parents' Motivation For Physical Activity And Their Beliefs, And Support Of Their Children's Physical Activity: A Cluster Analysis. Psychol Rep. 2015;117(1): 230-43.

43. Standage M, Duda JL, Treasure DC, et al. Validity, Reliability, and Invariance of the Situational Motivation Scale (SIMS) Across Diverse Physical Activity Contexts. J Sport Exerc Psychol. 2003;25(1):19-43.

\section{*Correspondence to}

Han-Hung Huang

Department of Physical Therapy

Angelo State University, Texas Tech University System 2601 W Ave N, San Angelo, TX 76904

USA

E-mail: hhuang@angelo.edu 